\title{
Supplementary Contents
}

\author{
A Succinct Synthesis of Bosentan by utilizing Glycol mono-THP ether \\ Chung-Ryul Lee; Sang Yeul Lee and Tae-gyu Nam* \\ Department of Pharmacy and Institute of Pharmaceutical Science and Technology \\ Hanyang University, Ansan, Gyeonggi-do 426-791, South Korea
}

S1: Experimental

S3: Selected NMR Spectra of Compounds: 13, 14, and 1 (Bosentan)

S6: HPLC traces of new compounds: 13 and $\mathbf{1 4}$ 


\section{Experimental}

General: Materials were purchased from commercial supplier and used without further purification. Progress of reaction was monitored by thin-layer-chromatography (TLC) using silica gel $\mathrm{F}_{254}$ plates. Purification of the products was performed using silica gel chromatography with indicated solvents. NMR spectra were obtained using a Bruker-250 spectrometer $400 \mathrm{MHz}$ for ${ }^{1} \mathrm{H}$ NMR and $100 \mathrm{MHz}$ for ${ }^{13} \mathrm{C}$ NMR and are reported as ppm from the internal standard tetramethylsilane (TMS). High-resolution mass spectra (HRMS) were obtained in Gyeonggi Institute of Science and Technology Promotion (GSTEP).

2-((Tetrahydro-2H-pyran-2-yl)oxy)ethan-1-ol (15). A stirred solution of ethylene glycol (10.0 mL, $118.5 \mathrm{mmol})$ and PPTS $(450 \mathrm{mg}, 1.8 \mathrm{mmol})$ in $\mathrm{CH}_{2} \mathrm{Cl}_{2}(250 \mathrm{~mL})$ at ambient temperature was treated dropwise with a solution of 3,4-dihydro-2H-pyran (DHP; $10.8 \mathrm{~mL}$, $179.0 \mathrm{mmol})$ in $\mathrm{CH}_{2} \mathrm{Cl}_{2}(50 \mathrm{~mL})$ over $1 \mathrm{~h}$. The reaction mixture was stirred for $18 \mathrm{~h}$ and washed with water and aqueous $10 \% \mathrm{NaCl}$. Resulting organic phase was dried over anhyd. $\mathrm{MgSO}_{4}$ and concentrated under reduced pressure to provide the crude product. Flash chromatography $\left(\mathrm{SiO}_{2}, 1: 1 \mathrm{EtOAc}\right.$ :hexanes) to provide 15 as a colorless oil $(8.8 \mathrm{~g}, 51 \%):{ }^{1} \mathrm{H}$ NMR (400 MHz, $\left.\mathrm{CDCl}_{3}\right) \delta$ 4.44-4.31 (m, 1H), $3.79(\mathrm{~m}, 1 \mathrm{H}), 3.68-3.48(\mathrm{~m}, 2 \mathrm{H}), 3.47-3.43$ (m, 1H), 3.39-3.31 (m, 2H), 3.28-3.320 (m, 1H), 1.63-1.41 (m, 2H), 1.38-1.19 (m, 4H); CAS Registry Number: 2162-31-4.

4-tert-Butyl- $N$-(5-(2-methoxyphenoxy)-6-(2-(tetrahydro-2H-pyran-2-yloxy)ethoxy)-2,2'bipyrimidin-4-yl)benzenesulfonamide (14). From (6): A solution of compound 15 (580 mg, $3.98 \mathrm{mmol})$ in anhydrous DMF $(20 \mathrm{~mL})$ at $0{ }^{\circ} \mathrm{C}$ was treated with $\mathrm{NaH}(60 \%$ in mineral oil, $191 \mathrm{mg}, 4.78 \mathrm{mmol}$, washed with hexanes). The resulting mixture was stirred at $0{ }^{\circ} \mathrm{C}$ for 30 min under Ar and treated with a solution of commercially available 6 (purchased from Rovathin, $1990 \mathrm{mg}, 3.79 \mathrm{mmol})$ in DMF $(19 \mathrm{~mL})$. The reaction mixture was stirred at $0{ }^{\circ} \mathrm{C}$ 
for $1 \mathrm{~h}$ and then at ambient temperature for $5 \mathrm{~h}$ under Ar. The reaction mixture was diluted with EtOAc $(500 \mathrm{~mL})$, washed with aqueous $1 \mathrm{~N} \mathrm{HCl}(3 \times 30 \mathrm{~mL})$, water $(3 \times 50 \mathrm{~mL})$ and saturated aqueous $\mathrm{NaCl}$, dried over anhyd. $\mathrm{MgSO}_{4}$, and concentrated under reduced pressure to provide the crude product. Flash chromatography $\left(\mathrm{SiO}_{2}, 3 \% \mathrm{MeOH}-\mathrm{CH}_{2} \mathrm{Cl}_{2}\right)$ to provide 14 as green foam $(1.64 \mathrm{~g}, 68 \%)$.

Bosentan (1). A solution of compound 14 (145 mg, $0.23 \mathrm{mmol})$ in $\mathrm{MeOH}(5 \mathrm{~mL})$ at ambient temperature was treated with pyridinium $p$-toluenesulfonate $(8 \mathrm{mg}, 0.032 \mathrm{mmol})$. The reaction mixture was stirred for $24 \mathrm{~h}$ under $\mathrm{Ar}$ and concentrated under reduced pressure. The residue was dissolved in EtOAc $(100 \mathrm{~mL})$, washed with water $(5 \times 10 \mathrm{~mL})$ and saturated aqueous $\mathrm{NaCl}$, dried over anhyd. $\mathrm{MgSO}_{4}$ and concentrated under reduced pressure to provide the crude product. Flash chromatography $\left(\mathrm{SiO}_{2}\right.$, gradient, 3 to $\left.10 \% \mathrm{MeOH}-\mathrm{CH}_{2} \mathrm{Cl}_{2}\right)$ to provide 1 as a white solid (107 mg, 84\%): ${ }^{1} \mathrm{H}$ NMR (400 MHz, $\left.\mathrm{CDCl}_{3}\right) \delta 9.24(\mathrm{~s}, 1 \mathrm{H}), 8.95$ $(\mathrm{d}, J=4.8 \mathrm{~Hz}, 2 \mathrm{H}), 8.41(\mathrm{~d}, J=6.8 \mathrm{~Hz}, 2 \mathrm{H}), 7.42-7.34(\mathrm{~m}, 3 \mathrm{H}), 7.02-6.98(\mathrm{~m}, 2 \mathrm{H}), 6.87(\mathrm{~d}$, $J=7.2 \mathrm{~Hz}, 1 \mathrm{H}), 6.75(\mathrm{t}, J=7.4 \mathrm{~Hz}, 1 \mathrm{H}), 4.76(\mathrm{~s}, 1 \mathrm{H}), 4.54-4.48(\mathrm{~m}, 2 \mathrm{H}), 3.81(\mathrm{~s}, 3 \mathrm{H}), 3.79-$ $3.74(\mathrm{~m}, 2 \mathrm{H}), 1.22(\mathrm{~s}, 9 \mathrm{H}) ;{ }^{13} \mathrm{C} \mathrm{NMR}\left(100 \mathrm{MHz}, \mathrm{CDCl}_{3}\right) \delta 161.2,157.6(2 \mathrm{C}), 157.0,155.1$, 151.6, 149.4, 145.4, 136.1, 129.3 (2C), 125.3 (3C), 125.1, 124.4, 121.3, 121.0, 118.7, 112.3, 71.3, 62.1, 55.8, 35.0, 30.9 (3C); CAS Registry Number: 147536-97-8. 
5-(2-Methoxyphenoxy)-6-(2-(tetrahydro-2H-pyran-2-yloxy)ethoxy)-4-amino-2,2'-bipyrimidine (13)

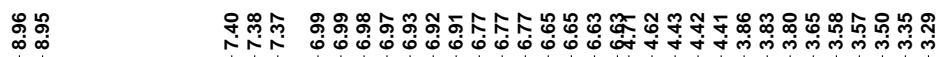

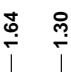<smiles>COc1ccccc1Oc1c(Cl)nc(-c2ncccn2)nc1OCC[OH+]</smiles>

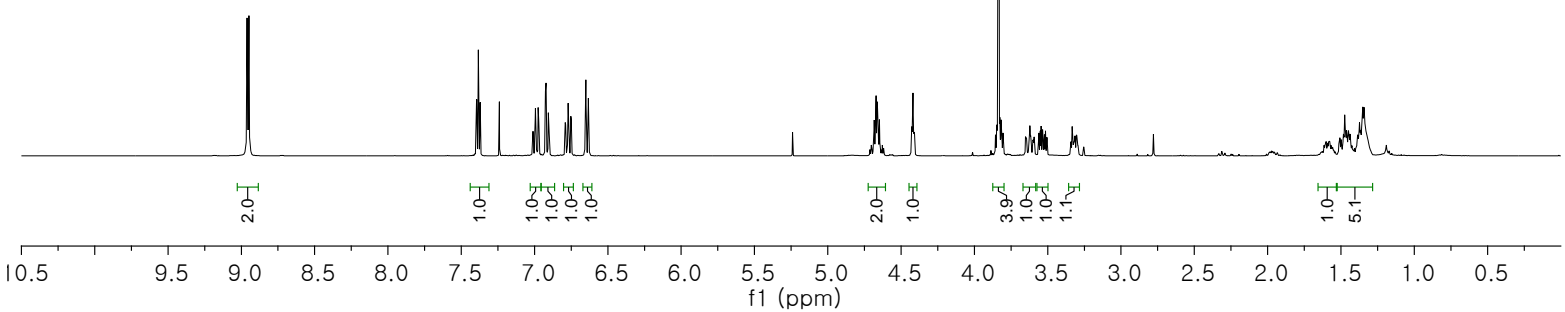

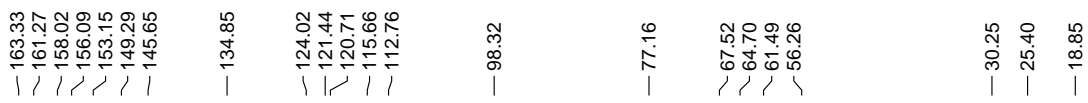<smiles>COc1ccccc1Oc1c(Cl)nc(-c2ncccn2)nc1OCC[OH+]</smiles>

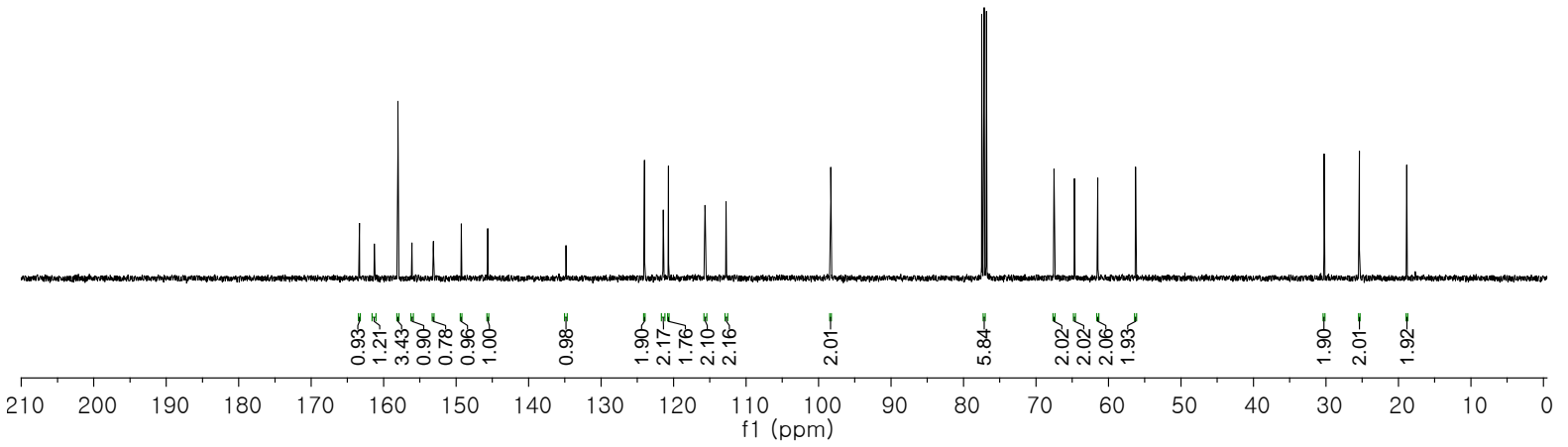


4-tert-Butyl-N-(5-(2-methoxyphenoxy)-6-(2-(tetrahydro-2H-pyran-2-yloxy)ethoxy)-2,2'-bipyrimidin-

4-yl)benzenesulfonamide (14)

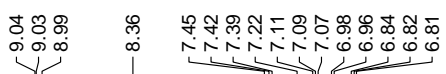

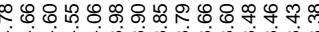

1

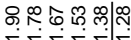<smiles>COc1ccccc1Oc1c(NS(=O)(=O)c2ccc(C(C)(C)C)cc2)nc(-c2ncccn2)nc1OCCF</smiles>

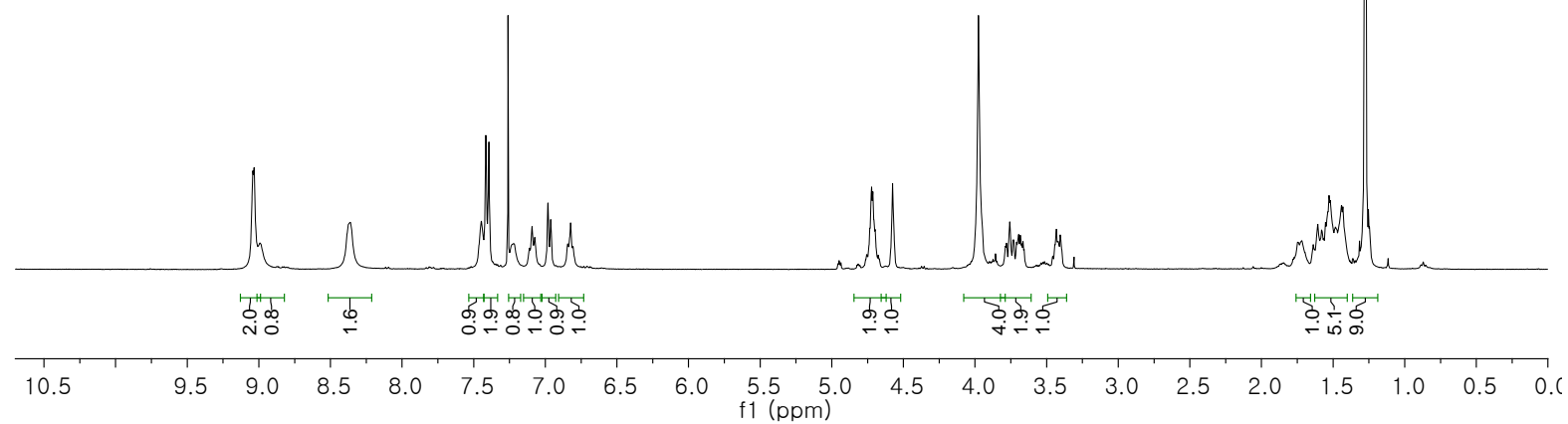<smiles>COc1ccccc1Oc1c(NS(=O)(=O)c2ccc(C(C)(C)C)cc2)nc(-c2ncccn2)nc1OCCF</smiles>

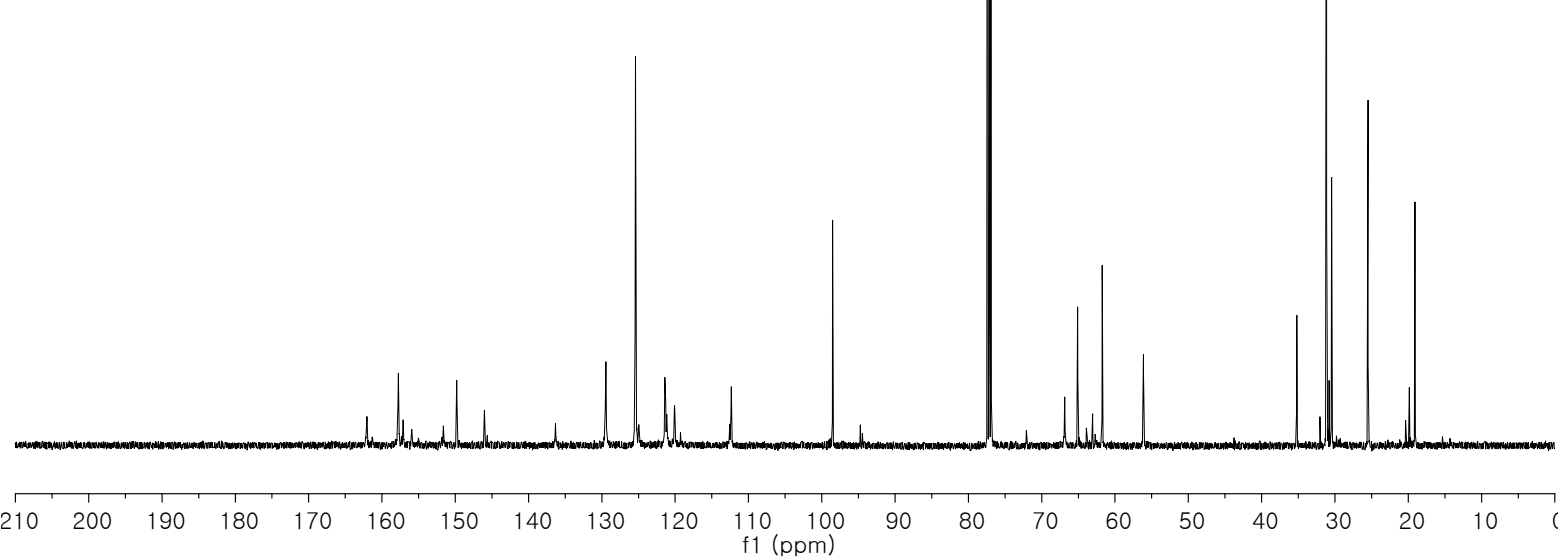




\section{Bosentan (1)}

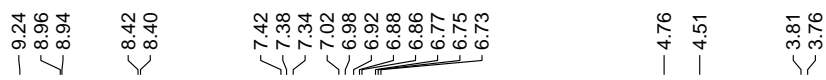<smiles>COc1ccccc1Oc1c(NS(=O)(=O)c2ccc(C(C)(C)C)cc2)nc(-c2ncccn2)nc1OCCO</smiles>

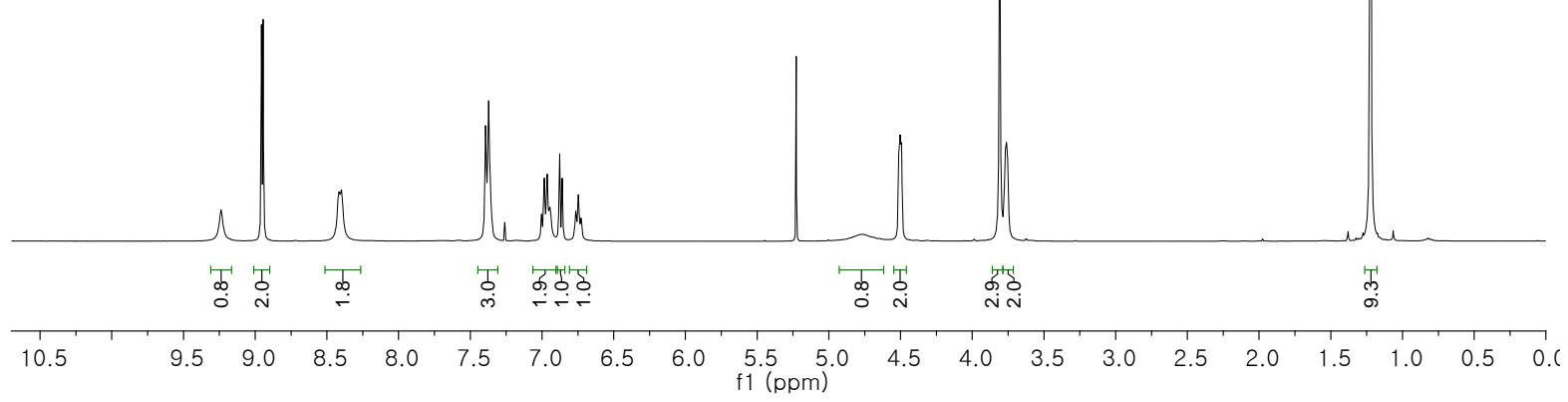

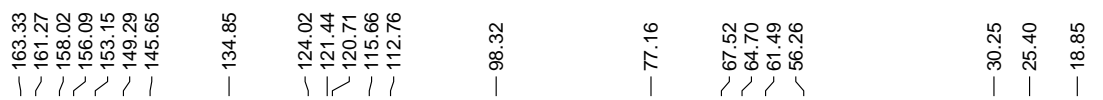<smiles>COc1ccccc1Oc1c(NS(=O)(=O)c2ccc(C(C)(C)C)cc2)nc(-c2ncccn2)nc1OCCO</smiles>

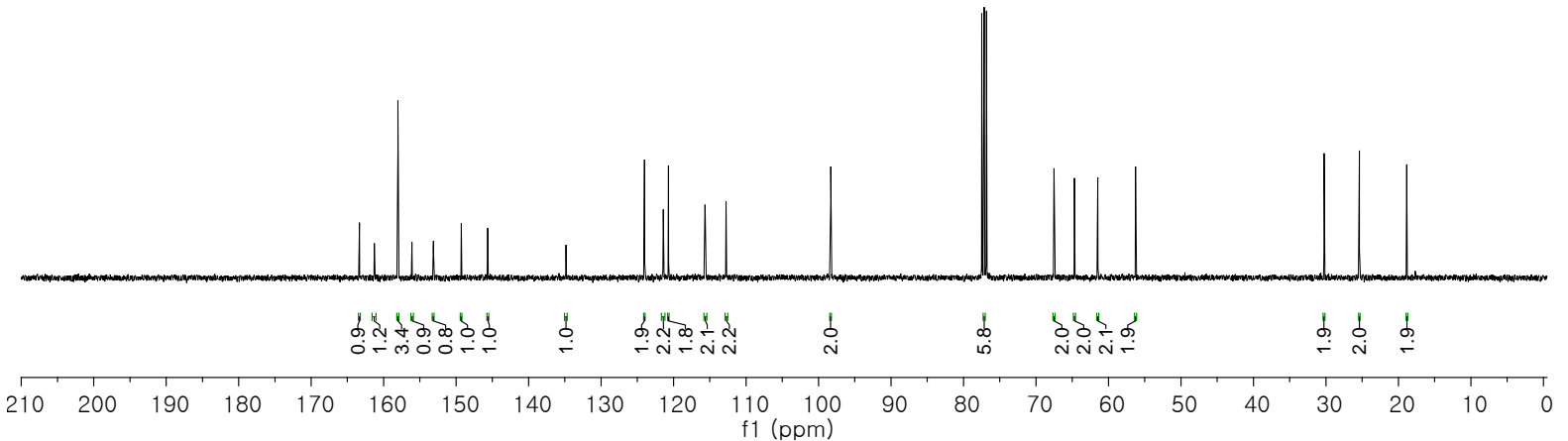


HPLC Trace of Compound $\mathbf{1 3}$
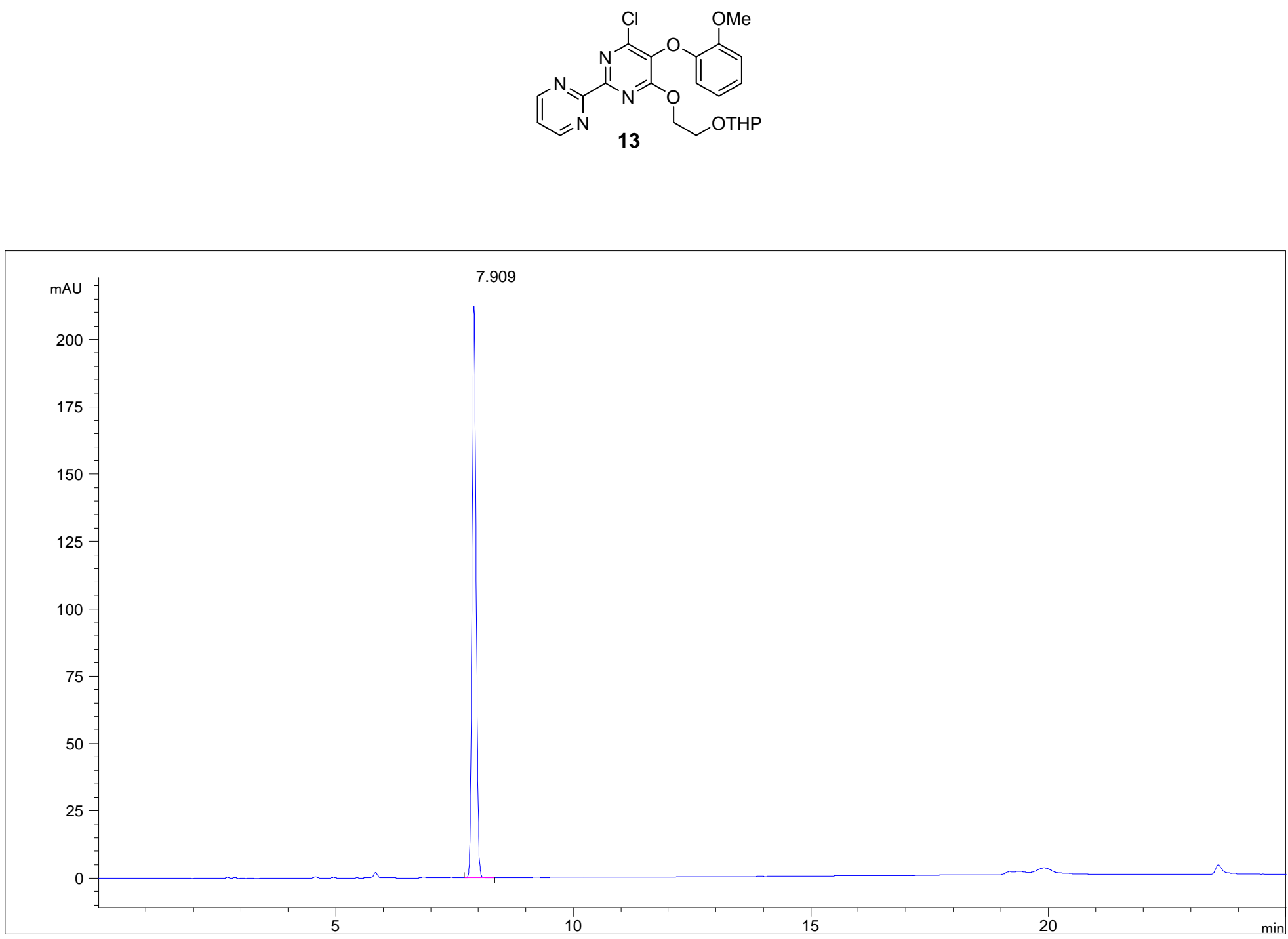

\begin{tabular}{|c|c|}
\hline Time (min) & solvent $\mathrm{A} \%$ \\
\hline 0 & 50 \\
\hline 20 & 100 \\
\hline 25 & 100 \\
\hline $\begin{array}{l}\text { A: acetonitrile }(0 . \\
\text { B: water }(0.1 \% \text { fo } \\
\text { Flow rate: } 1 \mathrm{~mL} / \mathrm{r} \\
\text { Detection wave } 1\end{array}$ & id) \\
\hline \multicolumn{2}{|c|}{ Column: INNO C18 [5 $\mu \mathrm{m}, 120 \dot{\mathrm{A}}, 4.6 \times 250 \mathrm{~mm}]$} \\
\hline
\end{tabular}


HPLC Trace of Compound 14
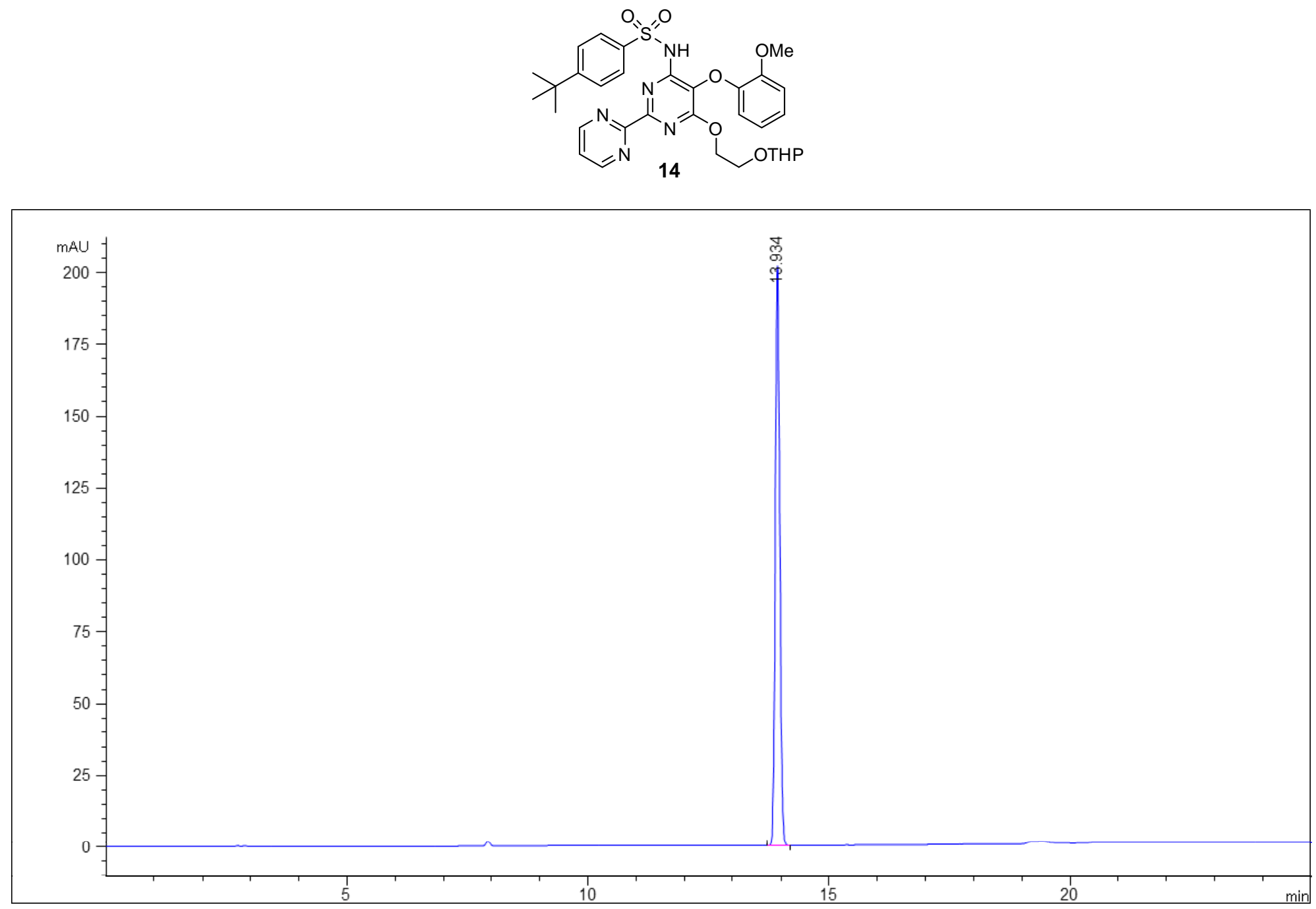

\begin{tabular}{|c|c|}
\hline Time $(\min )$ & solvent A \% \\
\hline 0 & 50 \\
\hline 20 & 100 \\
\hline 25 & 100 \\
\hline
\end{tabular}

A: acetonitrile $(0.1 \%$ formic acid $)$

$\mathrm{B}$ : water $(0.1 \%$ formic acid)

Flow rate: $1 \mathrm{~mL} / \mathrm{min}$

Detection wave length: $280 \mathrm{~nm}$

Column: INNO C18 [5 $\mu \mathrm{m}, 120 \dot{\mathrm{A}}, 4.6 \times 250 \mathrm{~mm}]$ 